\title{
Motivos da utilização frequente do serviço de urgência pelas pessoas idosas no contexto de uma unidade de saúde familiar
}

\begin{abstract}
Idalina Delfina Gomes ${ }^{1}$ e Alexandra Seara Almeida ${ }^{2}$
${ }^{1}$ Professora Doutora Coordenadora da Escola Superior de Enfermagem de Lisboa, Portugal | idgomes@esel.pt | https//orcid.org/0000-0003-2974-0734

${ }^{2}$ Enfermeira mestranda do $10^{\circ}$ Curso de Mestrado em Enfermagem na Área de Especialização em Enfermagem Médico-Cirúrgica, na Área de Intervenção em Enfermagem à Pessoa Idosa da Escola Superior de Enfermagem de Lisboa, Portugal | alexandraseara51@gmail.com | https://orcid.org/0000-0001-6528-2211

Resumo: A população a nível mundial encontra-se a envelhecer, o que leva a um aumento de doenças crónicas e a um aumento do risco de fragilidade, com implicações na utilização frequente do serviço de urgência pelas pessoas idosas, com impacto a nível social e da saúde o que deve constituir uma preocupação dos enfermeiros que precisam de intervir na promoção da saúde desta população. Objetivo: analisar os motivos da utilização frequente do serviço de urgência pelas pessoas idosas; Método: estudo descritivo qualitativo, com recurso a entrevista semiestruturadas e análise de conteúdo; participantes - 6 (pessoas idosas frágeis e familiares cuidadores); Resultados: constatou-se que a maioria dos motivos que levaram os participantes a recorrer ao serviço de urgência estavam relacionados com períodos de agudização da(s) doença(s) crónica(s) e as razões relacionadas com: a procura de cuidados especializados, referenciação por profissionais de saúde, medo de agravamento da situação de doença e dificuldade na acessibilidade à unidade de saúde. Conclusões: os enfermeiros dos cuidados de saúde primários precisam de apostar mais na vigilância da(s) doenças crónicas(s) e promover a literacia da pessoa idosa e do familiar cuidador para promover o Cuidado-de-SI.
\end{abstract}

Palavras-chave: Pessoa Idosa; Fragilidade; Utilizador Frequente; Serviço de Urgência.

Reasons for the Frequent Use of the Emergency Service by the Elderly in the Context of a Family Health Unit

\begin{abstract}
The world population is aging, which leads to an increase in chronic diseases and an increased risk of frailty, with implications for the frequent use of the emergency department by the elderly, with an impact on social and health levels, which should be a concern for nurses who need to intervene in promoting the health of this population; Goals: analyse the reasons for the frequent use of the emergency department by the elderly; Method: qualitative descriptive study, using semi-structured interviews and content analysis; participants - 6 (frail elderly and family caregivers); Results: it was found that most of the reasons that led the participants to make use of emergency department were related to periods of acute of the chronic disease(s), search for specialized care, referral by healthcare professionals, fear of worsening the disease and difficulty in accessing the primary healthcare facility; Conclusions: primary healthcare nurses need to invest more in the surveillance of chronic diseases(s) and promote the literacy of the elderly and the family caregiver to promote the Self-Care.
\end{abstract}

Keywords: Elderly; Frailty; Frequent User; Emergency Department.

\section{Introdução}

O envelhecimento populacional é uma realidade a nível mundial. Estima-se que em 2025 existirão em todo o mundo 1,2 mil milhões de pessoas idosas e destas, com ou mais de 80 anos constituirão um grupo etário de extrema importância. O aumento da esperança média de vida é um objetivo partilhado por inúmeras sociedades, contudo, este facto só se traduz numa conquista se for acompanhado por qualidade de vida. Neste sentido, as políticas de saúde devem incidir na promoção do envelhecimento ativo e saudável (WHO, 2020). Porém, constata-se que o envelhecimento populacional tem levado ao aumento da incidência e prevalência de doenças, de comorbilidades, das condições de fragilidade, assim como ao aumento dos índices de óbitos causados pelas doenças crónicas não transmissíveis (Observatório Português dos Sistemas de Saúde [OPSS], 2018). 
Desta forma, a população idosa é um dos grupos etários que mais recorre ao serviço de urgência (SU), tornando-se utilizadores frequentes do serviço de urgência (UFSU) o que faz alterar a capacidade e os cuidados no mesmo. Existem algumas definições para o conceito de UFSU, a que reúne maior consenso é a do utente que recorre ao SU, num período de um ano um mínimo de quatro vezes (Dufour et al., 2019). As pessoas idosas apresentam uma maior taxa de internamento hospitalar, risco de declínio funcional e morte após uma visita ao SU. Além disso, constata-se que muitos desses episódios são de utilização inadequada levando a um excessivo uso dos meios complementares de diagnósticos, de tratamento, e à superlotação do SU e ao aumento do tempo de espera, traduzindo-se num aumento dos custos com a saúde e num fardo para os sistemas de saúde (Observatório Português dos Sistemas de Saúde [OPSS], 2017). Conscientes desta problemática os enfermeiros da Unidade de Saúde Familiar X (USFX), e nomeadamente porque esta Unidade apresentava e uma taxa anual ajustada de episódios de urgência hospitalar de $62,39 \%$, perceberam a necessidade imperiosa de promover o Cuidado-de$\mathrm{Si}$ às pessoas Idosas, visando a prevenção e controlo da UFSU, indo ao encontro das suas reais necessidades e potencialidades.

Primeiramente há que identificar os motivos dessa UFSU. Contudo, na revisão da literatura efetuada, revelaram-se escassos os estudos qualitativos que incidem sobre os motivos da UFSU sob ponto de vista das pessoas idosas e familiares cuidadores (FC).

Assim, colocámos a seguinte questão de investigação: Quais os motivos que levam as pessoas idosas da USFX a serem UFSU?

\section{Metodologia}

Optou-se pela realização de um estudo qualitativo com recurso a entrevista semiestruturada, porque pretendíamos obter informações sobre o que pensam os sujeitos do estudo (Minayo \& Costa, 2018), ou seja perceber os motivos da UFSU. A pesquisa qualitativa está relacionada com a capacidade de compreensão do significado de um fenómeno e descrição pormenorizada dos contextos, sem dar relevância aos números "a quantidade é substituída pela intensidade, pela imersão profunda" (Taquette, 2016, p. 526). Deste modo, não existe verdade absoluta, uma vez que, se pretende compreender a complexidade de um fenómeno. A entrevista permite colher dados fornecidos pelos entrevistados, que estão relacionados com o conhecimento, atitudes, crenças e sentimentos (Batista et al., 2017). Assim, procurou-se responder às seguintes questões: Quais os motivos que levam as pessoas idosas da USFX a serem UFSU? As respetivas pessoas idosas e seus FC têm a perceção que são UFSU? Conhecem outros recursos na comunidade a que recorrer em situações de urgência? Este estudo tem por objetivo identificar os motivos da UFSU e face aos resultados, analisar como os enfermeiros da respetiva unidade podem ajudar a prevenir e controlar esta situação na USFX.

Participantes do estudo: três pessoas idosas e três FC. A escolha dos participantes da entrevista foi por conveniência, tendo em conta os critérios de inclusão: pessoas idosas (65 e mais anos) utentes da USFX; que após aplicação do instrumento de avaliação Mini Exame do Estado Mental não revelassem alterações cognitivas; pessoas idosas UFSU ou FC de pessoas idosas utentes da USFX que preencham os critérios acima referidos e cujas pessoas idosas não tivessem capacidade para responder.

\subsection{Procedimentos Metodológicos}

Os participantes foram selecionados pelos enfermeiros de família (EF), tendo em conta os critérios de inclusão acima referidos. Num primeiro contato o $\mathrm{EF}$, através de consulta ou telefone, abordou o entrevistado no sentido de o informar da existência do estudo, do respetivo objetivo e da extrema importância em participar. Segundo Batista et al. (2017) esta abordagem diminui o fator surpresa e dá confiança ao entrevistado. Posteriormente, foi contatado o entrevistado para confirmar se continuava interessado em participar na entrevista. Após aprovação combinou-se o dia e o local. 
No momento da entrevista a abordagem consistiu na apresentação do investigador, na explicitação da finalidade do estudo e do objetivo da entrevista. Foi igualmente explicado que a participação era voluntária, que seria assegurado anonimato, que a entrevista seria audiogravada e que a qualquer momento poderia desistir, sem sofrer qualquer tipo de coação. Antes de se iniciar a entrevista foi permitido o esclarecimento de dúvidas e solicitado consentimento informado e esclarecido. No decorrer da entrevista tentou-se proporcionar promover um ambiente harmonioso propício a expressão de sentimentos e emoções. O entrevistado pode escolher o locar da entrevista o que possibilitou uma maior segurança e abertura de emoções e sentimentos (especialmente quando a opção foi o domicílio). As entrevistas tiveram uma duração média de quinze minutos, uma vez que eram pessoas idosas frágeis. Foram realizadas entre os dias quatro a treze de dezembro de 2019. A utilização de entrevistas semiestruturadas foi facilitadora para redirecionar e focalizar a entrevista pela flexibilidade que permite. A mesma foi terminada de forma cordial, agradecendo a participação do entrevistado e mostrando disponibilidade para eventuais esclarecimentos que possam surgir.

Como foi referido, um dos critérios de inclusão consistia em identificar se existia alguma alteração do estado mental dos participantes, que comprometesse a informação fornecida pelos mesmos e quando assim fosse a entrevista era realizada aos FC. Deste modo, identificámos que dos seis participantes inicialmente referenciados três apresentaram alteração do estado mental, assim a entrevista foi realizada aos respetivos FC que demonstraram disponibilidade e desejo de participar.

A transcrição das entrevistas foi efetuada em ambiente silencioso, foram ouvidas inúmeras vezes e transcritas na integra, nomeadamente as pausas e a entoação da voz.

Os dados foram analisados seguindo os princípios de análise de conteúdo. Na pesquisa qualitativa, a análise de conteúdo, trata-se de uma descrição objetiva, sistemática, quantitativa do conteúdo extraído das comunicações e respetiva interpretação. Segundo (Bardin, 2016), a análise de conteúdo apresenta 3 etapas: pré-análise, exploração do material e por último tratamento dos resultados, inferência e interpretação. A pré-análise é uma fase em que se sistematizam as ideias iniciais, colocadas pelo quadro referencial teórico, de modo a elaborar um esquema compreensível que dê significado aos dados. $\mathrm{Na}$ exploração do material, codificam-se os dados, em que estes são transformados sistematicamente e agregados em unidades. Consiste na exploração do material com a definição de categorias e da identificação de unidades de registo. A categorização consiste na organização das categorias, onde estas e as subcategorias são explicadas, com base nas unidades de sentido identificadas anteriormente (Leite, 2017).

Neste estudo foram cumpridas as três etapas sugeridas por Bardin (2016). As categorias e as subcategorias foram definidas à posteriori, ou seja, de forma indutiva, a partir dos significados referidos pelos participantes de forma explícita. Assim, realizou-se uma divisão manual da informação recolhida das entrevistas em unidades manejáveis de síntese, na procura de padrões e de descoberta. Na categorização tivemos em conta os princípios da exclusividade entre categorias, da homogeneidade dentro das categorias e da objetividade entre os codificadores e a adequabilidade e pertinência aos conteúdos e objetivos (Bardin, 2016). A análise da informação recolhida, foi agrupada em categoria, subcategorias, unidades de registo e unidades de frequência. De forma a simplificar as unidades de registo foram codificadas em " $U$ " quando se referem a declarações prestadas pelos próprios utentes e em "F" quando as informações se referem aos FC. Para garantir a fiabilidade dos dados a análise de conteúdo foi aferida pelos dois investigadores.

\subsection{Principios Éticos}

A realização do estudo obteve parecer favorável da Comissão de Ética para a Saúde para a ARSLVT - Proc.111/CES/INV/2019. Todos os participantes foram informados sobre os objetivos do estudo e assinaram o consentimento informado. Garantiu-se o anonimato dos participantes e da instituição, bem como o sigilo e a confidencialidade das informações prestadas. 


\section{Resultados}

Os participantes foram seis (três pessoas idosas e três FC dos respetivos utentes). Em relação às pessoas idosas, um era do sexo masculino e dois do sexo feminino. As idades situavam-se entre os 70 e os 78 anos de idade. Após a aplicação do TFI pode-se concluir que os três utentes entrevistados eram todos pessoas idosas frágeis. Relativamente aos FC dois eram do sexo feminino e um do sexo masculino, dois destes eram os conjugues dos utentes e um deles era filha. Todos viviam com o respetivo utente.

Após a análise das entrevistas emergiram quatro categorias: Perceção de ser UFSU; Serviços que dão apoio ao SU, Situação de saúde/doença que levaram ao SU e Razões que levaram a recorrer ao SU.

Relativamente à categoria "Perceção de ser UFSU" nesta identificaram-se as subcategorias: Utilizador não frequente do SU e Utilizador frequente do SU. Na categoria "Serviços que dão apoio ao SU" evidenciaram-se as subcategorias: conhecimento e desconhecimento dos serviços de apoio. Em relação à categoria "Situação de saúde/doença que o levaram ao SU", surgiram as subcategorias: acidentes e agravamento da doença crónica e percebeu-se que quando eram UFSU por motivo de doença na maior parte das vezes (com quatro unidades de frequência) o motivo foi sempre o mesmo. A última categoria "Razões que levaram a recorrer ao SU" identificaram-se as subcategorias: Procura de cuidados especializados; Referência por profissionais de saúde; Medo de agravamento da situação de doença; Dificuldade na acessibilidade à USFX.

\section{Discussão dos Resultados}

Em relação à categoria "Perceção de ser UFSU", concluímos que apenas um dos seis participantes tinha a perceção de ser um UFSU, como refere F02 "Fui quatro vezes ao SU." Os restantes cinco participantes não tinham essa perceção. A referir, que após consentimento dos próprios, tinha sido primeiramente consultado o processo clínico, no sentido de confirmar se todos os utentes eram UFSU, tendo-se constado que sim (uma vez que este era um dos critérios de inclusão). Este resultado leva-nos a pressupor que os participantes podem não dar a devida importância quando recorrem ao SU. Este é um aspeto importante a ser aprofundado, na medida em que o enfermeiro pode informar os utentes e FC sobre os vários serviços que dão apoio ao SU, nomeadamente à Consulta Aberta Diária (CAD) da USFX, explicar a finalidade de cada serviço e as implicações e consequências quando o SU é utilizado sem ser para situações urgentes.

Relativamente à categoria "Serviços que dão apoio ao SU". Apenas o participante U4 é que afirmou que conhecia a USFX como serviço que dá apoio ao SU como nos referiu: "É só aqui. Já aqui venho há trinta anos", referindo-se à CAD da USFX. Esta dificuldade em identificar outros serviços que dão apoio ao SU, foi igualmente constatada na pesquisa efetuada, pelos autores Andrews \& Kass (2018). Deste modo, salienta-se a importância de os enfermeiros promoverem a sua função de educação e ensino para informar e esclarecer os utentes e FC sobre a existência dos diversos serviços que dão apoio ao SU, assim como a sua finalidade o que pode evitar os episódios frequentes de idas ao SU.

Na categoria "Situação de saúde/doença que o levaram ao SU" após a análise dos dados, constatou-se que a maior parte das situações de saúde que fizeram com que os participantes recorressem ao SU, estavam relacionados com períodos de agudização da doença(s) crónica(s), sobrepondo-se a situações associadas a acidentes, nomeadamente a quedas. As situações de doença mais frequentes estavam relacionadas com o sistema circulatório e cardíaco, úlcera varicosas, úlcera diabética, dor, sistema urinário e respiratório. Outro aspeto a salientar, é que os participantes do estudo, também referiram que as situações de saúde que os fizeram recorrer ao SU da última vez, eram os mesmos que das outras vezes, "Foram, sempre os mesmos..." (U05). Apenas um participante referiu que se dirigiu ao SU por motivos diferentes. 
O que nos leva a refletir na importância do papel do enfermeiro na promoção do Cuidadode-SI, tendo em conta o duplo sentido deste conceito: o de ajudar as pessoas idosas a gerir as situações de saúde doença, promovendo nelas o Cuidado-de-SI próprias ou capacitando o FC para assegurar o cuidado do seu familiar doente (Gomes, 2021).

Por último na categoria "Razões que levaram a recorrer ao SU" percebeu-se que um FC recorre ao SU com o seu familiar devido à dificuldade na acessibilidade à USFX, em que um FC verbalizou "(...) porque no Centro de Saúde não me atendem na altura própria. Tenho, tenho que ir para o hospital." (F02). Dois utentes e dois FC foram ao SU para terem acesso a cuidados especializados. Outras vezes são mesmos os profissionais de saúde (médico de família e assistentes em cuidados domiciliários) que os referenciam. Por outro lado, um FC considerou que os sintomas precisam de resposta urgente e referiu medo dos mesmos se agravem se não forem atendidos rapidamente como se pode constatar na seguinte frase significativa: "Bem eu só vou ao serviço de urgência quando efetivamente tenho mesmo medo que aconteça qualquer coisa mais grave." (F06). Estes resultados vão ao encontro do contatado por Coster et al. (2017), em que os resultados do estudo mostraram que os utentes consideravam que a sua condição exigia recursos e instalações especializadas, por desejarem ser atendidos rapidamente e pela dificuldade no acesso aos Cuidados de Saúde Primários (CSP). Os autores Kraaijvanger et al. (2016), salientaram que as razões se prendiam com medo de condições de risco de vida. Deste modo, torna-se primordial que os enfermeiros consigam desmitificar os preconceitos relacionados com o SU e sobre o funcionamento e atendimento da USFX, mais especificamente a CAD e promovam um acompanhamento de proximidade com as pessoas idosas que as ajude a gerir as suas situações de saúde/fragilidade/doença no seu quotidiano de Vida. Estas asserções têm implícito uma teoria holística do cuidado, remetendo um Modelo Centrado na Pessoa (McCance et al., 2020).

\section{Conclusões}

Ao longo do tempo tem-se verificado um aumento crescente da utilização frequente do SU com aumento dos custos e sobrecarga do serviço. Após a análise das entrevistas realizadas aos seis participantes, pode-se constar que se tratavam todos de pessoas Idosas frágeis em que a maioria não tinha a perceção que era um UFSU. Este facto tornase numa barreira para diminuir/controlar esta problemática.

Os participantes do estudo não identificaram a CAD, inserida na USFX, como sendo um dos serviços que dá apoio ao SU para situações urgentes. Por outro lado, constatou-se que a maioria dos motivos que levaram os participantes a recorrer ao SU estavam relacionados com períodos de agudização da(s) doença(s) crónica(s), sobrepondo-se aos motivos associados a acidentes, e as razões relacionadas com a procura de cuidados especializados; referenciação por profissionais de saúde; medo de agravamento da situação de doença e dificuldade na acessibilidade à USF. O que realça a importância dos profissionais de saúde e nomeadamente os enfermeiros dos CSP apostarem mais na prevenção e gestão da doença crónica e promover a literacia da pessoa idosa e do FC em parceria, por forma a promover o Cuidado-de-SI, para um desenvolvimento sustentável da saúde, em que a saúde/doença seja vista como um projeto em inter-relação com o projeto de vida e existência da pessoa (I. D. Gomes, 2019).

O recurso a análise de conteúdo realizada focada no emergir dos significados que os participantes dão aos fenómenos quando estão em estudo problemas emergentes, mas ocultos, contribui para mostrar a importância do desenvolvimento da pesquisa qualitativa.

Como limitação do estudo salienta-se o facto de não se ter conseguido incluir mais participantes, devido à dificuldade em identificar utentes como UFSU e as dificuldades colocadas pelas questões da pandemia, pelo que no futuro pensamos retomar o estudo. 


\section{Referências}

Andrews, H., \& Kass, L. (2018). Non-urgent use of emergency departments: populations most likely to overestimate illness severity. Internal and Emergency Medicine, 1-8. doi: ncbi.nlm.nih.gov/pubmed/29380133.

Bardin, L. (2016). Análise de conteúdo (1. ${ }^{\mathrm{a}}$ ed.). Edições 70.

Batista, E. C., Matos, L. A. L., \& Nascimento, A. B. (2017). A entrevista como técnica de investigação na pesquisa qualitativa. Revista Interdisciplinar Científica Aplicada, 11(3), 23-38. doi: file:///C:/Users/EU/Downloads/Aentrevistacomotcnicadeinvestigaonapesquisaqualitativa (1).pdf.

Coster, J. E., Turner, J. K., Bradbury, D., \& Cantrell, A. (2017). Why Do People Choose Emergency and Urgent Care Services? A Rapid Review Utilizing a Systematic Literature Search and Narrative Synthesis. Academic Emergency Medicine, 24(9), 1137-11-49. doi: ncbi.nlm.nih.gov/pmc/articles/PMC5599959/pdf/ACEM-24-1137.pdf.

Dufour, I., Chouinard, M.-C., Dubuc, N., Beaudin, J., Lafontaine, S., \& Hudon, C. (2019). Factors associated with frequent use of emergency-department services in a geriatric population: a systematic review. BMC Geriatrics, 19(185), 1-9. doi: bmcgeriatr.biomedcentral.com/track/pdf/10.1186/s12877-019-1197-9.

Gomes, I. (2021). Partnership of Care in the Promotion of the Care-of-the-Self: An Implementation Guide with Elderly People. Em J. Garcia-Alonso \& C. Fonseca (Eds.), Gerontechnology III: Contributions to the Third International Workshop on Gerontechnology, IWoG 2020 (pp. 345356). Évora: Lecture Notes in Bioengineering - Springer.

Gomes, I. D. (2019). Promover o Cuidado-de-Si: património da enfermagem para o desenvolvimento sustentado, bem-estar e saúde das populações. Revista Pensar Enfermagem, 23(2), 7-15.

Kraaijvanger, N., Rijpsma, D., Willink, L., Lucassen, P., Leeuwen, H. van, \& Edwards, M. (2016). Why patients self-refer to the Emergency Department: A qualitative interview study. Journal of Evaluation in Clinical Practice, 23, 593-598. doi: onlinelibrary.wiley.com/doi/epdf/10.1111/jep.12680.

Leite, R. F. (2017). Perspectiva da análise de conteúdo na pesquisa qualitativa: algumas considerações. Revista Pesquisa Qualitativa, 5(9), 539-551. doi: editora.sepq.org.br/rpq/article/view/129/98.

McCance, T., McCormack, B., Slater, P., \& McConnell, D. (2020). Examining the theoretical relationships between constructs in the person-centered practice framework: a structural equation model. BMC Health Services Research, 1-23. doi: 10.21203/rs.3.rs-22586/v1.

Minayo, M. C. S., \& Costa, A. P. (2018). Fundamentos teóricos das técnicas de investigação qualitativa. Revista Lusófona de Educação, 40, 139-153. doi: revistas.ulusofona.pt/index.php/rleducacao/article/view/6439.

Observatório Português dos Sistemas de Saúde [OPSS]. (2017). Viver em tempos incertos sustentabilidade e equidade na saúde. OPSS. doi: opss.pt/wpcontent/uploads/2018/06/Relatorio_Primavera_2017.pdf

Observatório Português dos Sistemas de Saúde [OPSS]. (2018). Meio caminho andando - Relatório primavera 2018. OPSS. doi: opss.pt/wp-content/uploads/2018/06/relatorio-primavera-2018.pdf.

Taquette, S. R. (2016). Análise de dados de pesquisa qualitativa em saúde. Atas CIAIQ 2016, 2, 524-533. doi: proceedings.ciaiq.org/index.php/ciaiq2016/article/view/790.

WHO. (2020). Decade of Healthy Ageing (2020-2030). doi: who.int/docs/default-source/decade-ofhealthy-ageing/final-decade-proposal/decade-proposal-final-apr2020en. pdf?sfvrsn=b4b75ebc. 